\title{
CHEMICAL COMPOSITION OF DUST EXPECTED FROM CONDENSATION MODELS
}

\author{
TETSUO YAMAMOTO \\ Institute of Space and Astronautical Science \\ Yoshinodai 3-1-1, Sagamihara \\ Kanagawa 229 \\ Japan
}

\begin{abstract}
This review examines to what degrees the present chemical equilibrium condensation models are effective in predicting chemical composition of grains observed in a variety of cosmic environments. The composition expected from the equilibrium calculations is reviewed separately for refractory (rocky and metallic) and volatile (icy) components. Comments are given on the limitation of the equilibrium calculations in predicting the grain composition. By taking cometary ice as a typical cosmic volatile condensate, it is pointed out that its composition is far from that expected from the equilibrium models. Theories on the formation of cometary volatiles are reviewed, and an observational clue helpful to testing the theories is pointed out. Discussion is given on the advantage for formation of organic materials from volatile solids.
\end{abstract}

\section{Introduction}

Condensation is an elementary process for formation of dust grains. Models of condensation of gases having various elemental composition provide a basis of a study of the chemical composition of grains formed in a variety of cosmic environments. This review concentrates on the composition as viewed from the condensation theory. Spectroscopic studies of the chemical composition are addressed to a review by Tielens in this volume.

The condensates are classified into refractories and volatiles. The refractory solids include rocky and metallic materials, whereas the volatiles are ices of various composition composed mainly of $\mathrm{H}, \mathrm{C}, \mathrm{N}, \mathrm{O}$ and $\mathrm{S}$. Organic materials are another important component of grains, which have similar elemental composition as volatiles, but are more refractory. Table 1 gives a rough idea on the elements relevant to the above classification of the grain materials. Condensation (or sublimation) temperatures mainly depend on the strength of the bond; volatiles have the weakest bond such as van der Waals or hydrogen bonds, whereas refractories have strong bonds such as valence or metallic bonds. Condensation temperatures of volatiles are lower than $100 \mathrm{~K}$ in general, whereas those of rocky and metallic grains are higher than $1000 \mathrm{~K}$ under typical pressures relevant to cosmic condensation. Table 1 also indicates that the abundances of $\mathrm{C}, \mathrm{N}$, and $\mathrm{O}$, the elements composing volatiles 413

A.C. Levasseur-Regourd and H. Hasegawa (eds.), Origin and Evolution of Interplanetary Dust, 413-420. (C) 1991 Kluwer Academic Publishers, Printed in Japan. 
and organics, are about ten times larger than those of $\mathrm{Si}, \mathrm{Mg}, \mathrm{Fe}$, and $\mathrm{Ni}$, the elements composing rocks and metals. This implies that ice and organics have potential to be as abundant as rocky and metallic solids or more.

TABLE 1. Properties of abundant elements

$$
\left(T=10 \sim 1000 \mathrm{~K}, P \sim 10^{-4} \mathrm{~atm}\right)
$$

\begin{tabular}{|c|c|c|c|c|}
\hline & volatility & phase & bond & abundance \\
\hline $\begin{array}{l}\mathrm{H} \\
\mathrm{He}\end{array}$ & very volatile & gas & & $10^{10}$ \\
\hline $\begin{array}{l}\mathrm{C} \\
\mathrm{N} \\
\mathrm{O}\end{array}$ & $\begin{array}{l}\text { volatile } \\
\text { refractory }\end{array}$ & $\begin{array}{c}\text { ice } \\
\text { organics }\end{array}$ & $\begin{array}{c}\text { van der Waals } \\
\text { H-bond } \\
\text { partially valence }\end{array}$ & $10^{7}$ \\
\hline $\begin{array}{c}\mathrm{Mg} \\
\mathrm{Si}\end{array}$ & refractory & rock & valence & $10^{6}$ \\
\hline $\begin{array}{l}\mathrm{Fe} \\
\mathrm{Ni}\end{array}$ & refractory & $\begin{array}{c}\text { metal } \\
\text { (+oxide, sulfide) }\end{array}$ & metallic & $10^{6}$ \\
\hline
\end{tabular}

\section{Refractory Composition}

Chemical equilibrium condensation calculations have been established for rocky and metallic grains. The equilibrium calculations of a gas of solar elemental composition were intensively done in connection with the explanation of the composition of minerals in meteorites (see review by Grossman and Larimer, 1974). Major condensates that the equilibrium theory predicts are $\mathrm{Mg}$-silicate such as pyroxene and olivine, and Fe-Ni alloy. Silicates are observed in many interstellar and solar system objects in both amorphous and crystalline forms (e.g. Tielens, in this volume). Whether amorphous or crystalline solid is formed depends on the kinetics of condensation such as a crystalline nucleation rate and a cooling rate of the grains in the environment where the grains condense (Seki and Hasegawa, 1981). On the other hand, iron grains have not been observed in interstellar space, but the presence of iron or magnetite is suggested by depletion of $\mathrm{Fe}$ in the gas of interstellar space (e.g. Whittet, 1984)

Equilibrium calculations for condensation of a gas of elemental composition other than solar have also been done (Gilman, 1969; see also Woolf, 1975). The composition of condensates depends crucially on the $\mathrm{C} / \mathrm{O}$ ratio of the vapor. For $C / O<1$ as in the case of the solar composition, $\mathrm{C}$ atoms are locked in $\mathrm{CO}$ gas, which is stable at high temperatures and low pressures, so no free carbon is available for condensation. For $C / O>1$, on the other hand, free carbon is available for condensation. In this case, major condensates are $\mathrm{C}$ and $\mathrm{SiC}$. The presence of carbonaceous grains are suggested in circumstellar and interstellar space (e.g. a review by Duley, 1988), and also presolar graphite grains are identified, though very minor, in meteorites (Amari et al., 1990). $\mathrm{SiC}$ is observed in circumstellar envelopes of carbon stars and planetary nebulae (e.g. Bode, 1988), and pre-solar SiC is detected in meteorites (Bernatowicz et al., 1987). 
The chemical equilibrium theory has limitation for predicting solid composition formed through non-equilibrium processes. For carbonaceous grains, PAH (Léger and Puget, 1984) and QCC (Sakata et al., 1984), which would be non-equilibrium products, have been proposed from a comparison of the laboratory spectra with the interstellar and circumstellar spectra. The possibility of formation of hydrocarbon grains in the case of $C / O>1$ is suggested from thermodynamic calculations, because of the presence of abundant hydrogen (see review by Salpeter, 1977). For siliceous grains, Wada et al. (in this volume) propose non-stoichiometric $\mathrm{SiO}$ solid trapping $\mathrm{H}_{2} \mathrm{O}$ molecules in it, which is formed by rapid cooling of $\mathrm{SiO}$ gas, as a candidate dust composition around protostars. It must be pointed out that the elementary processes for the formation of those non-equilibrium condensates have not yet been clarified.

\section{Volatile Composition}

Volatiles have not been detected in interplanetary dust, but have been observed in the dust in interstellar clouds, and in comets. Volatiles are an excellent probe for studying the origin and evolution of dust including refractories, since volatiles are sensitive to temperature and radiation in the environment where they condensed and have been placed. In the study of the interrelation between interplanetary and interstellar dust, a comet is a key object (e.g. Greenberg, 1982;1988), since a comet is one of the most volatile-rich and pristine objects in the solar system probably preserving interstellar dust at the time of the formation of the solar system, as well as one of the main sources of present interplanetary dust. Volatile composition of comets is inferred from the gaseous composition observed in the coma.

Table 2 summarizes candidate molecules composing cometary volatiles and their abundances observed in the comae of comets (Weaver, 1989). Although the abundance values are heavily weighted towards the results of comet Halley and have uncertainties, it is clear that both oxidized $\left(\mathrm{CO}, \mathrm{CO}_{2}\right)$ and reduced $\left(\mathrm{CH}_{4}, \mathrm{NH}_{3}, \mathrm{HCN}\right)$ species constitute cometary volatiles. Namely, it is a remarkable characteristic that the cometary volatiles are a mixture of reduced and oxidized compounds. It must be noted that the chemical condensation theory (e.g. Lewis, 1974) predicts reduced compounds. Cometary volatiles are a typical sample of non-equilibrium condensates. This implies that the equilibrium theory breaks down at low temperatures, and non-equilibrium consideration is required for volatiles, as expected from the fact that the time required to achieve chemical equilibrium increases exponentially as the temperature is lowered.

TABLE 2. Relative abundances of molecules in the comae of comets (from Weaver, 1989)

\begin{tabular}{cccccccc}
\hline \hline $\mathrm{H}_{2} \mathrm{O}$ & $\mathrm{CO}$ & $\mathrm{CH}_{4}$ & $\mathrm{CO}_{2}$ & $\mathrm{H}_{2} \mathrm{CO}$ & $\mathrm{NH}_{3}$ & $\mathrm{HCN}$ & $\mathrm{N}_{2}$ \\
\hline 1 & $0.02-0.07$ & $0.01-0.05$ & $\sim 0.03$ & $\leq 0.05$ & $0.003-0.02$ & $\sim 0.001$ & $\leq 0.001$ \\
\hline \hline
\end{tabular}

Two types of the theories have been proposed for the formation of cometary volatiles. The theory of the first type assumes that cometary volatiles are a mixture of materials condensed in the solar nebula and in the Jovian subnebulae from a gas whose composition was quenched at a high temperature (Prinn and Fegley, 1989; Fegley and Prinn, 1989). 
Another theory of this type assumes that cometary volatiles are clathrate hydrates formed in the solar nebula. (Lunine, 1989; Engel et al., 1990). On the other hand, the theory of the second type asserts that cometary volatiles had originally condensed in the interstellar cloud, and lost very volatile species in the solar nebula (Yamamoto et al., 1983, Yamamoto, 1985). Namely cometary volatiles are regarded as a sublimation residue of the interstellar ice. These two types of the theories are distinguished by whether cometary volatiles originates as the condensates in the solar nebula including the subnebulae of the Jovian planets, or as sublimation residues of interstellar ice. In the following, I will briefly describe the mixture model and the interstellar-ice residue model. More detailed discussion is given by recent reviews by Yamamoto (1990a, b).

The 'mixture model' is based on a chemical equilibrium calculation of the molecular composition in a gas of solar composition, with taking account of quenching. In this model, the solar nebula is assumed to be initially hot $(>1000 \mathrm{~K})$. In the course of the nebular cooling, the gaseous composition is quenched at a certain temperature called a quenching temperature, since the time scale for achieving the chemical equilibrium becomes much longer than the nebular dynamical time scale at low temperatures. Condensation of volatiles occurs in the quenched gas. For carbon compounds, for example, a ratio of $\mathrm{CO} / \mathrm{CH}_{4}$ is a measure of the redox state. In a chemical equilibrium scheme, $\mathrm{CO}$ is a dominant carbon species of the gas at high temperatures and low pressures, and $\mathrm{CH}_{4}$ is the dominant species at low temperatures and high pressures. Actually, however, the gaseous composition is fixed in the cooling at a certain temperature called a quenching temperature. In consequence, $\mathrm{CO}$ dominates $\mathrm{CH}_{4}$ even at low temperatures at the pressures of the solar nebula that Fegley and Prinn (1989) suppose. On the other hand, $\mathrm{CH}_{4}$ dominates $\mathrm{CO}$ at the pressures of the Jovian subnebulae, since the total pressure of their hy pothetical Jovian subnebulae is much higher than that of the solar nebula. Similar situation holds for $\mathrm{N}_{2}$-to- $\mathrm{NH}_{3}$ conversion: the abundances are $\mathrm{N}_{2} \gg \mathrm{NH}_{3}$ in the solar nebula, whereas $\mathrm{NH}_{3} \sim \mathrm{N}_{2}$ in the Jovian subnebulae. In this model, cometary volatiles are assumed to be a mixture of condensates formed under both conditions.

It must be pointed out that $\mathrm{H}_{2} \mathrm{O}$ ice condenses at $\sim 150 \mathrm{~K}$ under the solar nebula conditions of the pressure of $\sim 10^{-3}$ to $10^{-5}$ bar (Lewis and Prinn, 1980) assumed in this model, and at a higher temperature under the subnebula conditions.

In the 'interstellar-ice residue model', the formation process of cometary volatiles is divided roughly into two stages. The first is the interstellar cloud stage, with its fragment being the parent cloud of the solar nebula. At this stage, gaseous molecules in the cloud condense onto grain surfaces to form volatile mantles on them. Since the grain temperature in dense regions of the cloud is as low as $\sim 10 \mathrm{~K}$, the grains can be coated with volatile mantles composed of molecules up to very volatile ones except $\mathrm{H}_{2}$ and $\mathrm{He}$. The volatile mantles are composed of a mixture of reduced and oxidized species as characterized by the Greenberg particle (Greenberg, 1982). Note that the chemical composition of the gas and volatile mantles is far from that expected from the thermochemical equilibrium, since the chemistry prevailing in interstellar clouds is not thermal chemistry, but is based on ionmolecule reactions at low temperatures and densities. The second is the primordial solar nebula stage. In the outer solar nebula where cometary nucleus formed, the grains in the parent interstellar cloud would have survived, but their mantles lost highly volatile species from their composition, since the temperature of the solar nebula would have been higher 
than that of the interstellar cloud. According to this model, cometary nuclei are regarded as planetesimals formed from grains coated with volatile mantles of the sublimation residues.

This model assumes that each of the volatile species composing the ice sublimes independently, which is rather too simplified in view of recent sublimation experiments of ice mixtures (see Yamamoto (1990a, b) for the implications of these experiments). Another assumption concerns the volatile mantle composition in the interstellar cloud. Namely, this model adopts the interstellar molecule (gaseous) composition. The composition of the volatile mantle (i.e. solid composition) is not necessarily the same as the gaseous composition (e.g. d'Hendecourt et al., 1985). It should be pointed out that the study of the ice in interstellar clouds is closely connected with the study of the cometary volatiles and of the origin of interplanetary dust.

Under the assumptions stated above, this model places a constraint upon the temperature that cometary volatiles experienced as: $T_{\text {subl }}\left(\mathrm{N}_{2}\right) \sim 20 \mathrm{~K}<T<T_{\text {subl }}\left(\mathrm{CO}_{2}\right) \sim 70 \mathrm{~K}$ from a comparison of the abundances of cometary and interstellar molecules, where $T_{\text {subl }}$ is the sublimation temperature. Note that cometary volatiles have not experienced the temperatures higher than $\sim 70 \mathrm{~K}$ according to this model.

Crystalline structure of the ice is one of the keys to testing the two types of the theories described above. Laboratory experiments (e.g. Klinger (1990) for a review) show that the crystalline structure of ices depends on the temperature at which they condense. At low. pressures relevant to cosmic ices, the structure is amorphous when condensed at low temperatures, whereas it is crystalline when condensed at high temperatures. For $\mathrm{H}_{2} \mathrm{O}$ ice, the transition temperature is 130 to $140 \mathrm{~K}$. As stated previously, the mixture model predicts that the $\mathrm{H}_{2} \mathrm{O}$ ice condenses at about $150 \mathrm{~K}$ in the solar nebula, and at a higher temperature in the Jovian subnebulae. According to the interstellar-ice residue model, on the other hand, the ice have not experienced temperatures higher than $\sim 70 \mathrm{~K}$. Thus, the mixture model predicts crystalline ice, whereas the interstellar-ice residue model predicts amorphous ice, though there is a possibility of alteration of the crystalline structure after condensation, for example, by UV irradiation (Kouchi and Kuroda, 1990). The crystalline structure of fresh ice in the interior of a cometary nucleus is a key to determining which theory is plausible. Direct observations of the ice in the interior of the nucleus may be difficult at present, and we may have to wait for a mission to the nucleus. At present, the spin temperature derived from the observations of the ortho/para abundance ratio of $\mathrm{H}_{2} \mathrm{O}$ molecules observed in comets Halley and Wilson (Mumma et al., 1987; Larson et al., 1988) will provide a clue to infer the formation temperature (see Yamamoto, 1990a).

\section{Formation Conditions of Organic Matter}

Organic materials are present ubiquitously in space. In interstellar space, the presence of organic refractory grains was proposed by Greenberg (1971) as resulting from photoprocessing of ice, and is confirmed from IR observations (Tielens, in this volume). Furthermore molecules of organic composition occupy a fraction of the observed interstellar molecular species in a gas phase. In the solar system, organic materials are found in carbonaceous chondrites, interplanetary dust as tar balls (Bradley and Brownlee, 1986), and cometary dust as CHON particles (Kissel and Krüger, 1987), and are suggested to be present on the 
surfaces of icy satellites of the Jovian planets. In a gas phase, atmospheres of the Jovian planets and their satellites contain organic species.

In the formation of organic materials, a redox state is one of the important factors. Many experiments suggest that a reduced state provides more favorable conditions for forming organic materials than an oxidized state. It is interesting to compare the redox state of the gas and solid phases in space by noting molecular species composed of carbon, nitrogen, or both. The gas phase carbon and nitrogen chemistry indicates that $\mathrm{C}$ is mainly in the form of $\mathrm{CO}$, and $\mathrm{N}$ in the form of $\mathrm{N}_{2}$ in interstellar clouds (e.g. Irvine and Hjarmarson, 1983) and in the solar nebula (Prinn and Fegley, 1989; Fegley and Prinn, 1989). Namely, C and $\mathrm{N}$ are mainly in the oxidized state in the gas phase. The reduced state is realized only locally such as in the atmospheres of the Jovian planets, and in the Jovian subnebulae. On the other hand, volatile solid composition is not known well at present compared with the gaseous composition. For the volatile solid composition in solar nebula, we can refer to the composition of cometary volatiles. If cometary volatiles represent primitive solar nebula volatiles, their composition indicates that the solar nebula volatile solids are more reduced than the nebular gas as stated in $§ 3$. In interstellar clouds, on the other hand, recent progress in infrared observations of dense clouds reveals the presence of $\mathrm{H}_{2} \mathrm{O}, \mathrm{CO}$ ices, and suggests $\mathrm{CH}_{3} \mathrm{OH}$, and $\mathrm{NH}_{3}$ as the ice composition (Tielens, in this volume). Figure 1 shows equilibrium temperatures of ices which condense from a gas of the interstellar molecule composition (Yamamoto et al., 1983), and is calculated based on the simplified assumption as stated in $§ 3$. It is clearly seen that the reduced compounds such as hydrocarbons, nitriles and ammonia condense more easily than the oxidized compounds such as $\mathrm{CO}$ and $\mathrm{N}_{2}$. These lines of results indicate that reduced compounds tend to be concentrated more in the volatile solids, and suggest that the solid phase provides more favorable conditions for the formation of precursors of organic materials than the gaseous phase.

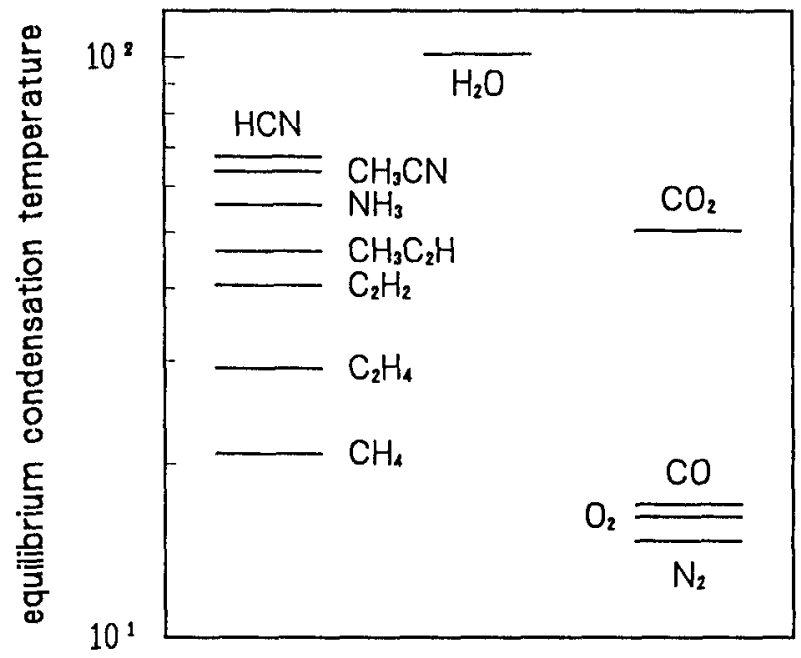

Figure 1. Equilibrium condensation temperatures (in K) of a gas of the interstellar molecule composition for the gas (mainly of $\mathrm{H}_{2}$ ) density of $10^{5} \mathrm{~cm}^{-3}$. The species of the left side are reduced ones, and those in the right side are oxidized ones. 


\section{Acknowledgments}

This work was partly supported by the Special Research Project on Evolution of Matter, University of Tsukuba.

\section{References}

Amari, S., Anders, E., Virag, A, and Zinner, E. (1990): 'Interstellar graphite in meteorites', Nature 345, 238-240.

Bernatowicz, T., Fraundorf, G., Ming, T., Anders, E., Wopenka, B., Zinner, E., and Fraundorf, P. (1987): 'Evidence for interstellar $\mathrm{SiC}$ in the Murray carbonaceous meteorites', Nature 330, 728-723.

Bradley, J.P. and Brownlee, D.E. (1986): 'Cometary particles: Thin sectioning and electron beam analysis', Science 231, 1542-1544.

Bode, M.F. (1988): 'Observations and modelling of circumstellar dust', Dust in the Universe, eds. M.E. Bailey and D.A. Williams, Cambridge University Press, Cambridge, pp. 73-102.

d'Hendecourt, L.B., Allamandola, L.J., and Greenberg, J.M. (1985): 'Time dependent chemistry in dense molecular clouds I. Grain surface reactions, gas/grain interactions and infrared spectroscopy', Astron. Astrophys. 152, 130-150.

Duley, W.W. (1988): 'Models of interstellar grains', Dust in the Universe, eds. M.E. Bailey and D.A. Williams, Cambridge University Press, Cambridge, pp. 209-218.

Engel, S., Lunine, J.I., and Lewis, J.S. (1990): 'Solar nebula origin for volatiles in Halley's comet', Icarus 85, 380-393.

Fegley, B., Jr., and Prinn, R.G. (1989): 'Solar nebula chemistry: Implications for volatiles in the solar nebula', The Formation and Evolution of Planetary Systems, eds. H.A. Weaver, and L. Danley, Cambridge Univ. Press., Cambridge, pp. 171-211.

Gilman, R.C. (1969): 'On the composition of circumstellar grains', Astrophys. $J$ 155, L185-L187.

Grossman, L. and Larimer, J.W. (1974): 'Early chemical history of the solar system', Rev. Geophys. Space Phys. 42, 71-101.

Greenberg, J.M. (1971): 'The chemical and physical properties of interstellar dust', Molecules in the Galactic Environment, eds. M.A. Gordon and L.E. Snyder, John Wiley \& Sons, New York, pp. 94-124.

Greenberg, J.M. (1982): 'What are comets made of? A model based on interstellar dust', Comets, ed. L.L. Wilkening, Univ. Arizona Press, Tucson, pp. 131-163.

Greenberg, J.M. (1988): 'The interstellar dust model of comets: post Halley', Dust in the Universe, eds. M.E. Bailey and D.A. Williams, Cambridge University Press, Cambridge, pp. 121-143.

Irvine, W.M. and Hjarmarson, $\AA$. (1983): 'Comets, interstellar molecules, and the origin of life', Cosmochemistry and the Origin of Life, eds. C. Ponnamperuma, D. Reidel, Dordrecht, pp. 113-142.

Klinger, J. (1990), 'Physical properties of frozen volatiles - Their relevance to the study of comet nuclei', 'Comets in the Post-Halley Era', eds. R. Newburn and J. Rahe, Kluwer Academic Publishers, in press. 
Kissel, J and Krüger, F.R. (1987): 'The organic component in dust from Comet Halley as measured by the PUMA mass spectrometer on board Vega' Nature 326, 755-760.

Kouchi, A. and Kuroda, T. (1990): 'Amorphization of cubic ice by ultraviolet radiation', Nature 344, 134-135.

Larson, H.P., Weaver, H.A., Mumma, M.J., and Drapatz, S. (1988): 'Airborne infrared spectroscopy of Comet Wilson (1986l) and comparison with Comet Halley', Astrophys. $J$. 338, 1106-1114.

Lewis, J.S. (1974): 'The temperature gradient in the solar nebula', Science 186, 440-443.

Lewis, J.s., and Prinn, R.G. (1980): 'Kinetic inhibition of $\mathrm{CO}$ and $\mathrm{N}_{2}$ reduction in the solar nebula', Astrophys. J. 238, 357-364.

Léger, A. and Puget, J.L. (1984): 'Identification of the 'unidentified' IR emission features of interstellar dust?', Astron. Astrophys. 137, L5 - L8.

Lunine, J.I. (1989): 'Primitive bodies: Molecular abundances in Comet Halley as probes of cometary formation environment', The Formation and Evolution of Planetary Systems, eds. H.A. Weaver, L. Danley, and F. Paresce, Cambridge Univ. Press., Cambridge, pp. 213-242.

Mumma, M.J., Weaver, H.A., and Larson, H.P. (1987): 'The ortho-para ratio of water vapor in comet $\mathrm{P} /$ Halley', Astron. Astrophys. 187, 419-424.

Prinn, R.G., and Fegley, B., Jr. (1989): 'Solar nebula chemistry: Origin of planetary, satellite, and cometary volatiles', Origin and Evolution of Planetary and Satellite Atmospheres, eds. S. Atrea, J. Pollack, and M. Matthews, Univ. Arizona Press, Tucson, pp. 78-136.

Sakata, A., Wada, S., Tanabé, T., and Onaka, T. (1984): 'Infrared spectrum of the laboratory-synthesized quenched carbonaceous composite (QCC): Comparison with the infrared unidentified emission bands', Astrophysical J. Lett. 287, L51 - L54.

Salpeter, E.E. (1977): 'Formation and destruction of dust grains', Ann. Rev. Astron. Astrophys. 15, 267-293.

Seki, J. and Hasegawa, H. (1981): 'Origin of amorphous interstellar ice grains', Prog. Theor. Phys. 66, 903-912.

Weaver, H.A. (1989): 'The volatile composition of comets', Highlights Astron. 8, 387-393.

Whittet, D.C.B. (1984): 'Interstellar grain composition: A model based on elemental depletions', Mon. Not. R. Astron. Soc. 210, 479-487.

Woolf, N.J. (1975): 'Circumstellar dust', Dusty Universe, eds. G.B. Field and A.G.W. Cameron, Neale Watson Academic Publications, Inc., New York, pp. 59-87.

Yamamoto, T. (1985): 'Formation environment of cometary nuclei in the primordial solar nebula', Astron. Astrophys. 142, 31-36.

Yamamoto, T. (1990a): 'Chemical theories on the origin of comets', 'Comets in the PostHalley Era', eds. R. Newburn and J. Rahe, Kluwer Academic Publishers, in press.

Yamamoto, (1990b): 'The origin of comets as viewed from the gaseous composition', Primitive Solar Nebula and Origin of the Planets, eds. H. Oya, K. Nakazawa, and H. Mizutani, Terra Publishing Company, Tokyo, in press.

Yamamoto, T., Nakagawa, N., and Fukui, Y. (1983): 'The chemical composition and thermal history of the ice of a cometary nucleus', Astron. Astrophys. 122, 171-176. 